\title{
Efectos de las Experiencias Adversas Temprana y Tardía en las Características Morfométricas y Estereológicas de la Glándula Suprarrenal de Ratas Sprague Dawley Sometidas a Estrés Durante su Vida Adulta
}

\author{
Effects of Early and Late Adverse Experiences in Morphometric and Stereological Characteristics \\ of the Adrenal Gland of Sprague Dawley Rats Subjected to Stress during Adulthood
}

\author{
Bélgica Vásquez ${ }^{*, * *}$; Cristian Sandoval ${ }^{* * *}$; Carla Sanhueza ${ }^{* * *}$; Jaime Silva ${ }^{* * * * *} \&$ Mariano del Sol ${ }^{* * * *}$
}

VÁSQUEZ, B.; SANDOVAL, C.; SANHUEZA, C.; SILVA, J. \& DEL SOL, M. Efectos de las experiencias adversas temprana y tardía en las características morfométricas y estereológicas de la glándula suprarrenal de ratas Sprague Dawley sometidas a estrés durante su vida adulta. Int. J. Morphol., 31(1):15-22, 2013.

RESUMEN: La ruptura temprana del vínculo materno y el aislamiento social son variables que están involucradas con los comportamientos social y emocional y también con el aumento de la ansiedad, especialmente en situaciones estresantes. Sin embargo, no se dispone de investigaciones que expliquen los cambios morfológicos de la glándula suprarrenal (GSR). Por lo anterior, el objetivo de este trabajo fue conocer experimentalmente, a través del modelo de alteración del vínculo social temprano madre-cría y alteración del vínculo social tardío por aislamiento, el efecto sobre las características morfométricas y estereológicas de la GSR, en ratas de la cepa Sprague Dawley sometidas a estrés crónico intermitente en la vida adulta. Utilizamos 35 ratas hembras recién nacidas, distribuidas en grupos de 7, en condiciones de lactancia y alimentación estandarizadas. Los grupos experimentales fueron expuestos a experiencias adversas temprana (E1), tardía (E2), temprana-tardía (E3) y posteriormente, sometidas a estrés crónico intermitente en la adultez. Se aisló la GSR izquierda de cada animal determinando características morfométricas y parámetros estereológicos. El peso absoluto fue mayor en los grupos control C2 y grupo experimental (E1). El número de células por mm³ , el porcentaje de tejido glandular y la densidad de superficie en la zona fascicular de la GSR fue menor en el grupo E3. Las características estereológicas de la GRS en ratas, no sólo pueden ser afectadas por la exposición al estrés en la adultez, sino que también las experiencias adversas temprana y/o tardía juegan un rol importante en los cambios de los parámetros morfológicos cuantitativos observados en esta glándula.

PALABRAS CLAVE: Rata; Estrés; Experiencia adversa; Glándula suprarrenal.

\section{INTRODUCCIÓN}

La literatura señala una relación entre el estrés y la actividad del eje hipotálamo-hipofisiario-adrenal (HHA). Genera diversas alteraciones en las características morfológicas de la corteza de la glándula suprarrenal (GSR), lo cual también estaría en estrecha relación con la síntesis y secreción de glucocorticoides que median la respuesta fisiológica al estrés.

El concepto de estrés hace referencia a una respuesta no específica del cuerpo a cualquier amenaza o fuerza externa, real o percibida, que gatilla el "Síndrome de adaptación general" en el organismo, (Ziegler \& Herman, 2002;
Goldstein \& Kopin, 2007), siendo el eje HHA uno de los principales elementos participantes. Las neuronas del núcleo hipofisiotrópico en el núcleo paraventricular del hipotálamo (NPH), secretan hormona liberadora de corticotropina y vasopresina a la circulación portal de la eminencia media. Estas hormonas de liberación actúan sobre la hipófisis anterior para promover la secreción de la hormona adrenocorticotrópica (ACTH) en la circulación sistémica. ACTH actúa sobre la corteza suprarrenal, en la zona fasciculada, para producir hormonas glucocorticoides (corticosterona en ratas y cortisol en humanos) (Herman $e t$ al., 1996; Ulrich-Lai et al., 2006).

* Universidad de Tarapacá, Arica, Chile.

** Doctorado en Ciencias Morfológicas, Universidad de La Frontera, Chile.

*** Universidad de La Frontera. Alumnos Tesistas, Universidad de La Frontera, Chile.

***** Facultad de Medicina, Universidad de La Frontera, Chile.

Financiado por Proyecto DI11-0054 de la Dirección de Investigación de la Universidad de La Frontera, Temuco, Chile. 
VÁSQUEZ, B.; SANDOVAL, C.; SANHUEZA, C.; SILVA, J. \& DEL SOL, M. Efectos de las experiencias adversas temprana y tardía en las características morfométricas y estereológicas de la glándula suprarrenal de ratas Sprague Dawley sometidas a estrés durante su Vida adulta. Int. J. Morphol., 31(1):15-22, 2013.

Chrousos (2009), señaló que en respuesta al estrés se activan distintas funciones del sistema nervioso central, entre ellas, la facilitación de la excitación, el estado de alerta, la vigilancia, cognición, atención y agresión. Por otro lado, genera la inhibición de las funciones vegetativas (por ejemplo: la reproducción, alimentación o crecimiento), y activación de los circuitos de retroalimentación negativa. También se ha asociado a la aparición de trastornos psiquiátricos y/o somáticos complejos, tales como ansiedad, depresión, insomnio, dolor crónico y síndromes de fatiga, obesidad, síndrome metabólico, hipertensión esencial, diabetes tipo 2, aterosclerosis con sus secuelas cardiovasculares y osteoporosis, así como la respuesta inflamatoria autoinmune y trastornos alérgicos (Chrousos \& Kino, 2007).

Nadal \& Armario (2010) señalaron que los efectos del estrés se encuentran relacionados con las características del estímulo estresor utilizado, es decir, la naturaleza del estímulo (físico o psicosocial), así como su intensidad y duración. Otro aspecto importante a considerar es la imprevisibilidad (imposibilidad de anticipación) y la ausencia del control sobre el estímulo (es decir, poner fin o disminuir su intensidad, duración o frecuencia).

Por otra parte, se han relacionado los efectos estresantes de una experiencia adversa temprana con la conducta que se adoptará durante la vida adulta. La ruptura del vínculo primario en ratas produce disrupción del mecanismo de escape (Ojea et al., 2010), altera el comportamiento social así como la emotividad en la edad adulta, pero no necesariamente la función cognitiva (Toth et al., 2008), además de una reducción significativa de la conducta exploratoria y cambios morfológicos neuronales a nivel frontal asociados a disminución del árbol dendrítico y aumento en la actividad del eje HHA (Pascual, 2002).

Así mismo, se ha señalado que la interrupción temprana del vínculo social madre-cría y un posterior aislamiento social en ratas, induciría a un mayor perfil ansiogénico durante estados de estrés en la vida adulta; además, luego de un período posterior de resocialización no se lograría recuperar el deterioro neuronal generado por el aislamiento, ni normalizar los niveles de ansiedad, con respecto al grupo control (Pascual et al., 2003). Este aumento de la ansiedad, según Bledsoe et al. (2011), estaría mediada por un incremento de los receptores tipo 2 del factor liberador de corticotropina en el núcleo dorsal del rafe.

La privación social es una de las condiciones experimentales que se ha utilizado para generar experiencias adversas tardías en modelos animales. Cruz (2003), observó que la privación social por un periodo de 15 días en ratas adultas, genera mayores niveles de ansiedad en el laberinto elevado en cruz y agresión en el modelo intrusoresidente. Malkesman et al., 2006, señalaron que el estrés crónico de 8 días de aislamiento ejerce influencia en las hormonas del eje HHA, con un aumento en los niveles plasmáticos de la corticosterona.

Por otro lado, Pellegrini et al. (1998), señalaron la existencia de un vínculo funcional entre las GSR y el estrés crónico inducido por inmovilización, en ratas machos adultas. El peso promedio de las GSR de las ratas intervenidas con estrés fue sólo ligeramente mayor que la del grupo control, hubo incremento de corticosterona plasmática e histológicamente, a nivel de corteza suprarrenal, se evidenció un aumento en el espesor de la zona fasciculada.

En un estudio realizado por Alario et al. (1987), ratas Wistar fueron sometidas a estrés crónico por ruido. Los resultados indicaron una disminución en la ingesta de alimento y del peso corporal. Se observó también una reducción del peso absoluto $(\mathrm{mg})$, pero no del peso relativo $(\mathrm{mg} /$ $\mathrm{g}$ de peso corporal) de las GSR, con respecto a los controles. Por otra parte, tampoco encontraron diferencias en el contenido de ADN adrenal absoluto $(\mu \mathrm{g})$, sugiriendo que el número de células no se ve afectado por estrés por ruido. Sin embargo, el alto contenido relativo de ADN adrenal ( $\mu \mathrm{g} / \mathrm{mg}$ gland.) en el grupo estresado indicó un tamaño más pequeño de dichas células. A partir de lo anterior, se propone que el bajo peso suprarrenal y el pequeño tamaño de las células en las GSR en las ratas estresadas por ruido, podría ser debido a un menor tamaño de los animales mediante la reducción de ganancia de peso corporal, en comparación con las ratas control.

Ulrich-Lai et al., establecieron que el estrés crónico aleatorio en ratas provoca una reducción de la ganancia de peso corporal y propicia el aumento de peso de la GSR. Demostraron además, que el estrés crónico variable se asocia a un aumento en los niveles plasmáticos de corticosterona, lo que induce un estado de hiperplasia en la zona glomerular y en la parte externa de la zona fasciculada, e hipertrofia en la parte interna de las zonas fasciculada y medular.

Koko et al. (2004), indicaron los efectos del estrés agudo por exposición al calor sobre las GSR de ratas. Observaron un incremento significativo de los niveles plasmáticos de las hormonas ACTH y corticosterona. En estas condiciones, la masa absoluta y relativa de la GSR disminuyeron significativamente, así como la masa absoluta de la corteza suprarrenal en comparación con los controles, probablemente como consecuencia de la reducción de todas las zonas corticales, especialmente la zona fasciculada. La fibrosis intersticial estaba presente en las 
VÁSQUEZ, B.; SANDOVAL, C.; SANHUEZA, C.; SILVA, J. \& DEL SOL, M. Efectos de las experiencias adversas temprana y tardía en las características morfométricas y estereológicas de la glándula suprarrenal de ratas Sprague Dawley sometidas a estrés durante su Vida adulta. Int. J. Morphol., 31(1):15-22, 2013.

zonas corticales complejas así como en la médula. La densidad del volumen de la médula adrenal aumentó significativamente, y la de la corteza disminuyó. No hubo diferencias significativas en el $\mathrm{Vv}$ y $\mathrm{Nv}$ de la zona fasciculada entre los dos grupos de ratas.

Recientemente, Petrovic-Kosanovic et al. (2012), encontraron resultados similares a los descritos por Koko et al., utilizando el mismo modelo animal. Sin embargo, estos autores no observaron diferencias significativas en el Vv de la médula y corteza adrenal entre los grupos control y experimental. El Nv de la zona fasciculada del grupo experimental fue significativamente menor al del grupo control.

Milosevic et al. (2005), utilizaron un modelo de estrés crónico en ratas hembras sometidas a luz constante, durante 95 días. Los resultados obtenidos demostraron que la exposición crónica provoca una disminución tanto del peso corporal, como del peso relativo y absoluto de la GSR. También observaron un incremento significativo de los volúmenes absoluto y relativo de la zona fasciculada, citoplasmas con zonas más claras, junto con un aumento del volumen celular y núcleos en dicha zona, comparado con ratas controles que se mantuvieron con ciclo día-noche de 12 horas luz/12 horas oscuridad. Como conclusión, los autores proponen que la exposición prolongada a la luz activaría los sistemas de estrés y consecuentemente aparecería hipertrofia en las células de la zona fasciculada, junto con un aumento en los niveles de corticosterona.

Si bien es cierto, se han realizado estudios respecto de cómo afecta el estrés, tanto agudo como crónico en la vida adulta y sus consecuencias en la morfología de la GSR de ratas, no existen investigaciones que entreguen información sobre el efecto de las experiencias adversas temprana y/o tardía en las características morfológicas de la GSR de ratas, cuando éstas son sometidas a estrés durante su vida adulta. Por lo anterior, el objetivo de este trabajo fue conocer experimentalmente, a través del modelo de alteración del vínculo social temprano madre-cría y alteración del vínculo social tardío por aislamiento, el efecto sobre las características morfométricas y estereológicas de la GSR en ratas de la cepa Sprague Dawley sometidas a estrés crónico intermitente en la vida adulta.

\section{MATERIAL Y MÉTODO}

Se utilizaron 35 ratas hembras albinas de la cepa Sprague Dawley, provenientes de la Unidad de Cirugía Experimental del Doctorado en Ciencias Morfológicas de la Facultad de Medicina de la Universidad de La Frontera. Las ratas recién nacidas fueron separadas en 5 grupos, cada uno con siete ratas asignadas aleatoriamente. Durante toda la experiencia las condiciones ambientales fueron controladas respecto a temperatura, ruido ambiental y ciclo día-noche de 12 horas luz/12 horas oscuridad. Las jaulas contaron con un bebedero graduado para el agua y un recipiente metálico para el alimento.

Los grupos fueron distribuidos de la siguiente forma:

Grupo control 1 (C1), grupo sin experiencias adversas temprana ni tardía, sin exposición a estrés crónico intermitente (utilizado como control morfológico normal de la GSR): Ratas recién nacidas con 23 días de lactancia, fueron separadas de su madre y mantenidas en jaula bajo condiciones de interacción social normal, agua y alimento (pellet) ad libitum durante toda la experiencia (116 días).

Grupo control 2 (C2), grupo sin experiencias adversas temprana ni tardía, expuesto a estrés crónico intermitente: Ratas recién nacidas con 23 días de lactancia, fueron separadas de su madre y mantenidas en jaula bajo condiciones de interacción social normal, agua y alimento (pellet) ad libitum, por un período de 110 días.

Grupo experimental 1 (E1), grupo con experiencia adversa temprana, expuesto a estrés crónico intermitente: Ratas recién nacidas con 18 días de lactancia, fueron separadas de su madre y mantenidas en jaula bajo condiciones de interacción social normal, agua y alimento (pellet) ad libitum, por un período de 110 días.

Grupo experimental 2 (E2), grupo con experiencia adversa tardía, expuesto a estrés crónico intermitente: Ratas recién nacidas con 23 días de lactancia, fueron separadas de su madre y mantenidas en jaula bajo condiciones de interacción social normal, agua y alimento (pellet) $\mathrm{ad}$ libitum, por un periodo de 80 días. Posteriormente, fueron colocadas en jaulas individuales impidiendo todo tipo de interacción social, hasta completar los 110 días. En este período se les ofreció agua y alimento (pellet) ad libitum.

Grupo experimental 3 (E3), grupo con experiencia adversa temprana y tardía, expuesto a estrés crónico intermitente: Ratas recién nacidas con 18 días de lactancia, fueron separadas de su madre y mantenidas en jaula bajo condiciones de interacción social normal, agua y alimento (pellet) ad libitum por un periodo de 80 días. Posteriormente, fueron colocadas en jaulas individuales impidiendo todo tipo de interacción social hasta completar los 110 días. En este período también tuvieron acceso a agua y alimento (pellet) ad libitum. 
VÁSQUEZ, B.; SANDOVAL, C.; SANHUEZA, C.; SILVA, J. \& DEL SOL, M. Efectos de las experiencias adversas temprana y tardía en las características morfométricas y estereológicas de la glándula suprarrenal de ratas Sprague Dawley sometidas a estrés durante su Vida adulta. Int. J. Morphol., 31(1):15-22, 2013.

Completados los 110 días, los integrantes de los grupos C2, E1, E2 y E3 fueron mantenidos individualmente con la finalidad de someterlos a estrés crónico intermitente. Para ello, durante 6 días consecutivos, las ratas fueron privadas de alimento por 20 horas diarias. Luego por 2 horas, en presencia de un estímulo visual (luz roja), se les proporcionó $50 \mathrm{~g}$ de pellet y $50 \mathrm{~g}$ de Quaker Quadritos®, junto a $200 \mathrm{ml}$ de agua (etapa de anticipación). Al término de éste periodo fueron retirados el alimento y el agua, para ser medidos. Posteriormente, durante dos horas más, se les proporcionó nuevamente $50 \mathrm{~g}$ de pellet y $50 \mathrm{~g}$ de Quaker Quadritos ${ }^{\circledR}$, junto a $200 \mathrm{ml}$ de agua, pero ésta vez en presencia de un estímulo estresor (tail pinch) (Katz \& Roth, 1979), (etapa estrés crónico intermitente). Finalizado este periodo, fue retirado el estímulo estresor y cuantificado el consumo de alimento y agua. A seguir, se les permitió beber agua ad libitum por 20 horas más para comenzar nuevamente con la etapa de anticipación.

A los 116 días post natal, las ratas fueron sacrificadas mediante dislocación cervical, para extraer la GSR izquierda de cada animal, las cuales fueron disecadas mesoscópicamente bajo lupa 10X. Se determinaron el alto, ancho y grosor de cada glándula con un paquímetro digital marca Stainless Hardened (0-150 mm), el peso en una balanza analítica marca A\&D Orion HR 120 (0,1 mg hasta $120 \mathrm{~g}$ ) y el volumen a través del método de Scherle (1970).

Para el estudio estereológico, se seleccionaron las GSR del lado izquierdo de las cinco primeras ratas de cada grupo, las que fueron fijadas en formalina tamponada al $10 \%$, por 24 horas, deshidratadas e incluidas en parafina. Obtenidos los tacos, se realizaron cortes de $4 \mu \mathrm{m}$ de grosor en un micrótomo (Microm HM325). En cada glándula se realizaron 5 cortes para luego ser teñidos con H-E. Se observaron 5 campos de cada corte, 125 en total. Las láminas fueron observadas en un microscopio óptico Olympus ${ }^{\circledR}$ modelo CX31, con cámara marca Moticam ${ }^{\circledR}$ modelo 480. Las imágenes fueron proyectadas en un monitor de pantalla plana marca Sony®. Para realizar la estereología se usó el test multipropósito M42.
Los parámetros estereológicos medidos en la zona fasciculada de la GSR fueron: densidad de número (Nv), densidad de volumen (Vv) y densidad de superficie (Sv). Para el análisis estadístico se utilizó el programa Stata versión 11.0 y se aplicó la prueba ANOVA. Se utilizó un nivel de significación de 5\% para las pruebas estadísticas. Una vez obtenidos estos datos, se calcularon el promedio, la desviación estándar (DE), error estándar (EE), coeficiente de variación (CV) y coeficiente de error (CE).

\section{RESULTADOS}

Morfometría de la glándula suprarrenal. Las medidas morfométricas de alto, ancho, grosor, peso y volumen promedio de las GSR de los distintos grupos experimentales, se muestra en la Tabla I. El peso absoluto de la GSR aumentó en los grupos $\mathrm{C} 2$ y E1, comparados con el grupo C1 y E2; sin embargo, las diferencias no fueron significativas ( $p>0,05)$. El volumen de la GSR fue levemente superior en todos los grupos experimentales, comparado con el $\mathrm{C} 1$, sin encontrar diferencias significativas entre ellos $(\mathrm{p}>0,05)$.

\section{Estereología de la glándula suprarrenal.}

La densidad de número promedio de células en la zona fasciculada fue mayor en el grupo $\mathrm{C} 1$ con 7,20 x 105/ $\mathrm{mm}^{3}(\mathrm{DE} 1,32)$ y menor en el grupo experimental E3, con $5,50 \times 10^{5} / \mathrm{mm}^{3}$ (DE 1,22) (Fig. 1). En la Tabla II se muestra el análisis estadístico de la Nv promedio de células en la zona fasciculada, según grupo en estudio.

La densidad de volumen promedio de células en la zona fasciculada fue mayor en el grupo experimental C2 con un $10,69 \%$ (DE 2,00) y menor en el grupo experimental E3 con un 8,34\% (DE 2,06) (Fig. 2). En la Tabla III se muestra el análisis estadístico de la Vv promedio de células en la zona fasciculada, según grupo en estudio.

Tabla I. Medidas morfométricas promedio de las glándulas suprarrenales de ratas hembras Sprague Dawley, sometidas a experiencias adversas temprana y tardía y posterior estrés crónico intermitente.

\begin{tabular}{lccccc}
\hline Grupos & Alto $(\mathbf{m m})$ & Ancho $(\mathbf{m m})$ & Grosor $(\mathbf{m m})$ & Peso $(\mathbf{g})$ & Volumen $\left.\left._{(\mathbf{m m}}\right)^{\mathbf{3}}\right)$ \\
\hline C1 & $4,6071 \pm 0,2611$ & $6,1886 \pm 0,1610$ & $3,2457 \pm 0,1602$ & $0,0513 \pm 0,0043$ & $0,0350 \pm 0,0053$ \\
C2 & $4,6786 \pm 0,3418$ & $6,1300 \pm 0,2409$ & $3,0486 \pm 0,1139$ & $0,0528 \pm 0,0075$ & $0,0373 \pm 0,0068$ \\
E1 & $4,6671 \pm 0,3266$ & $6,1300 \pm 0,2909$ & $2,7114 \pm 0,2371$ & $0,0531 \pm 0,0046$ & $0,0381 \pm 0,0041$ \\
E2 & $5,0514 \pm 0,3777$ & $6,2057 \pm 0,2781$ & $3,6300 \pm 1,7992$ & $0,0494 \pm 0,0042$ & $0,0389 \pm 0,0116$ \\
E3 & $4,6343 \pm 0,2669$ & $5,9914 \pm 0,2422$ & $3,0457 \pm 0,1591$ & $0,0487 \pm 0,0049$ & $0,0359 \pm 0,0051$
\end{tabular}




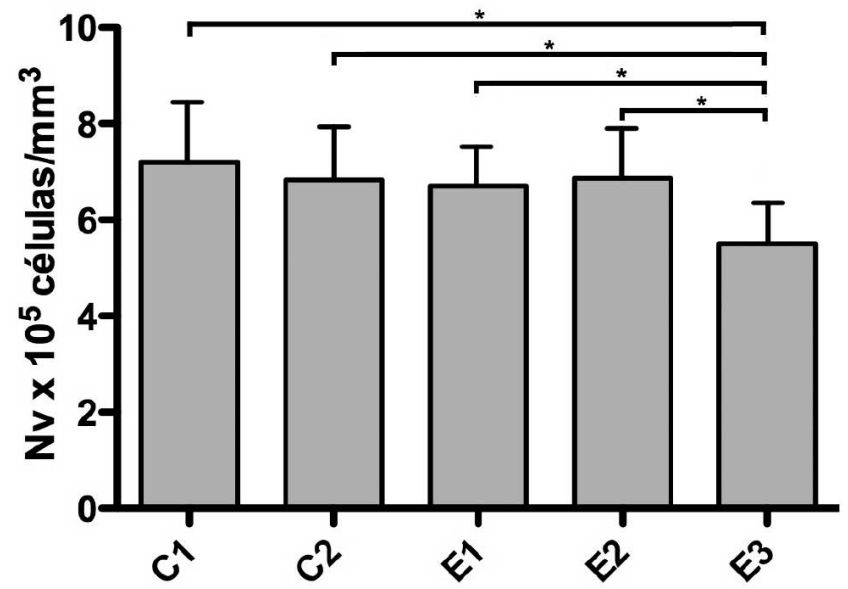

Fig. Densidad de número promedio de células glandulares de la zona fasciculada de la GSR de ratas hembras Sprague Dawley sometidas a experiencias adversas temprana y tardía y posterior estrés crónico intermitente.

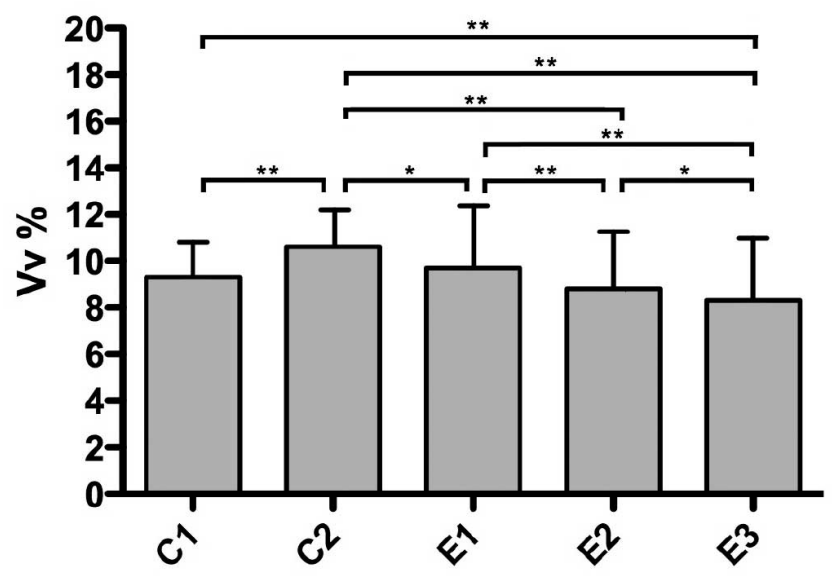

Fig. 2. Densidad de volumen promedio de células glandulares de la zona fasciculada de la GSR de ratas hembras Sprague Dawley sometidas a experiencias adversas temprana y tardía y posterior estrés crónico intermitente.

Tabla II. Análisis estadístico de la Nv de las células en la zona fasciculada de la GSR de ratas hembras Sprague Dawley sometidas a experiencias adversas temprana y tardía y posterior estrés crónico intermitente.

\begin{tabular}{|c|c|c|c|c|c|c|c|c|}
\hline NV & $\begin{array}{c}\text { Promedio } \\
\text { (células } / \mathrm{mm}^{3} \text { ) }\end{array}$ & $\mathrm{DE}$ & $\begin{array}{c}\text { Mínimo } \\
\text { (células/mm³) }\end{array}$ & $\begin{array}{c}\text { Máximo } \\
\text { (células } / \mathrm{mm}^{3} \text { ) }\end{array}$ & $\mathrm{EE}$ (células $/ \mathrm{mm}^{3}$ ) & $\begin{array}{l}\mathrm{CV} \\
(\%)\end{array}$ & $\begin{array}{l}\mathrm{CE} \\
(\%)\end{array}$ & $\begin{array}{l}\text { Valor } \\
\text { de p }\end{array}$ \\
\hline $\mathrm{C} 1$ & $7,20 \times 10^{5}$ & 1,32 & $5,76 \times 10^{5}$ & $8,74 \times 10^{5}$ & $0,59 \times 10^{5}$ & 18,31 & 8,19 & 0,9606 \\
\hline $\mathrm{C} 2$ & $6,83 \times 105$ & 1,22 & $5,68 \times 10^{5}$ & $8,13 \times 10^{5}$ & $0,54 \times 10^{5}$ & 17,78 & 7,95 & 0,9914 \\
\hline E1 & $6,71 \times 105$ & 1,18 & $5,85 \times 10^{5}$ & $8,13 \times 10^{5}$ & $0,53 \times 10^{5}$ & 17,64 & 7,89 & 0,7464 \\
\hline E2 & $6,86 \times 10^{5}$ & 1,13 & $5,82 \times 10^{5}$ & $8,19 \times 10^{5}$ & $0,51 \times 10^{5}$ & 16,46 & 7,36 & 0,5763 \\
\hline E3 & $5,50 \times 105$ & 1,22 & $4,25 \times 10^{5}$ & $7,01 \times 10^{5}$ & $0,54 \times 10^{5}$ & 22,11 & 9,89 & 0,9863 \\
\hline
\end{tabular}

Tabla III. Análisis estadístico de la Vv de las células en la zona fasciculada de glándula suprarrenal de ratas hembras sometidas a experiencias adversas temprana y tardía y posterior estrés crónico intermitente.

\begin{tabular}{ccccccccc}
\hline Vv & Promedio $\%$ & DE & Mínimo \% & Máximo \% & EE \% & CV $(\%)$ & CE $(\%)$ & Valor de p \\
\hline C1 & 9,33 & 1,60 & 7,62 & 10,95 & 0,72 & 17,17 & 7,68 & 0,9880 \\
C2 & 10,69 & 2,00 & 8,70 & 13,10 & 0,89 & 18,69 & 8,36 & 0,7688 \\
E1 & 9,77 & 2,10 & 7,35 & 11,96 & 0,94 & 21,56 & 9,64 & 0,9831 \\
E2 & 8,90 & 1,56 & 7,22 & 10,55 & 0,70 & 17,59 & 7,87 & 0,8462 \\
E3 & 8,34 & 2,06 & 5,98 & 10,82 & 0,92 & 24,72 & 11,05 & 0,8253 \\
\hline
\end{tabular}

Tabla IV. Análisis estadístico de la Sv de las células en la zona fasciculada de glándula suprarrenal de ratas hembras sometidas a experiencias adversas temprana y tardía y posterior estrés crónico intermitente.

\begin{tabular}{ccccccccc}
\hline Sv & $\begin{array}{c}\text { Promedio } \\
\left(\mathrm{mm}^{2} / \mathrm{mm}^{3}\right)\end{array}$ & DE & $\begin{array}{c}\text { Mínimo } \\
\left(\mathrm{mm}^{2} / \mathrm{mm}^{3}\right)\end{array}$ & $\begin{array}{c}\text { Máximo } \\
\left(\mathrm{mm}^{2} / \mathrm{mm}^{3}\right)\end{array}$ & $\begin{array}{c}\text { EE } \\
\left(\mathrm{mm}^{2} / \mathrm{mm}^{3}\right)\end{array}$ & CV (\%) & CE $(\%)$ & Valor de p \\
\hline C1 & 62,60 & 16,36 & 45,84 & 83,17 & 7,32 & 26,15 & 11,70 & 0,9431 \\
C2 & 71,75 & 13,83 & 56,25 & 85,97 & 6,18 & 19,24 & 8,60 & 0,9500 \\
E1 & 65,78 & 15,43 & 47,87 & 82,92 & 6,90 & 23,38 & 10,45 & 0,8764 \\
E2 & 68,44 & 15,20 & 50,79 & 85,71 & 6,80 & 22,13 & 9,90 & 0,3531 \\
E3 & 52,87 & 15,00 & 34,67 & 69,33 & 6,71 & 28,40 & 12,70 & 0,6620 \\
\hline
\end{tabular}


VÁSQUEZ, B.; SANDOVAL, C.; SANHUEZA, C.; SILVA, J. \& DEL SOL, M. Efectos de las experiencias adversas temprana y tardía en las características morfométricas y estereológicas de la glándula suprarrenal de ratas Sprague Dawley sometidas a estrés durante su Vida adulta. Int. J. Morphol., 31(1):15-22, 2013.

La densidad de superficie promedio de células en la zona fasciculada fue mayor en el grupo experimental C2, con $71,75 \mathrm{~mm}^{2} /$ $\mathrm{mm}^{3}(\mathrm{DE} 13,83)$ y menor en el grupo experimental E3, con 52,83 $\mathrm{mm}^{2} / \mathrm{mm}^{3}$ (DE 15,00) (Fig. 3). En la Tabla IV se muestra el análisis estadístico de la $\mathrm{Sv}$ promedio de células en la zona fasciculada, según grupo en estudio.

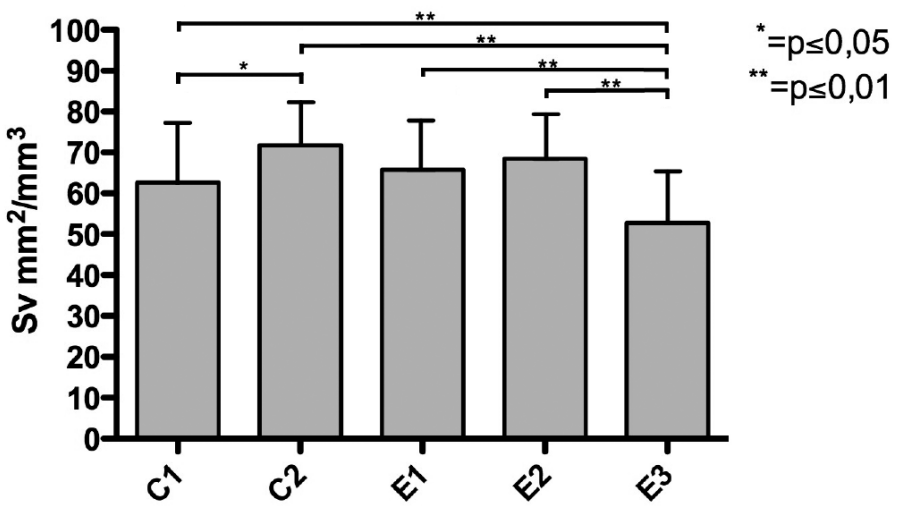

Fig. 3. Densidad de superficie promedio de células glandulares de la zona fasciculada de la GSR de ratas hembras Sprague Dawley sometidas a experiencias adversas temprana y tardía y posterior estrés crónico intermitente.

\section{DISCUSIÓN}

La literatura señala variadas investigaciones que abordan la relación entre la situación de estrés y sus consecuencias morfológicas en la GSR, siendo la rata el modelo animal más utilizado (Pellegrini et al.; Milosevic et al.; Ulrich-Lai et al. y PetrovicKosanovic et al.). Sin embargo, son escasos los estudios que relacionan las experiencias adversas temprana y tardía con cambios morfométricos y estereológicos en esta glándula.

Pellegrini et al., señalaron que en GSR de ratas adultas sometidas a estrés crónico intermitente por inmovilización, no hubo aumento significativo en el peso medio. Por el contrario, UlrichLai et al., indicaron aumentos significativos en el peso absoluto y relativo en las GSR de ratas sometidas a estrés crónico variable. En el presente estudio, hubo aumento del peso absoluto de la GSR en el grupo C2, aunque no fue significativo en comparación con el resto de los grupos, lo que concuerda con las observaciones de Pellegrini et al. Este grupo control, sólo fue sometido a estrés crónico intermitente, sin exponerlo a experiencias adversas previamente, a diferencia del grupo E1 que, aunque también presentó un leve aumento de peso absoluto en la GSR, si fue sometido a experiencias adversas tempranas.

Por el contrario, Milosevic et al., identificaron una reducción del 39\% del peso absoluto y un 32\% del peso relativo de la GSR en ratas sometidas a estrés crónico por exposición a luz cons- tante. Si bien en los grupos E2 y E3 se encontró una disminución del peso de las GSR comparada con los grupos controles, éstas no fueron estadísticamente significativas, por lo que es de suponer que las diferencias observadas por estos autores, podrían deberse a una mayor duración en el tiempo de exposición al estímulo estresor de su modelo.

En la literatura consultada respecto a los cambios estereológicos de la GSR en los distintos modelos de estrés aplicados en rata, se pueden observar resultados muy diversos. Alario et al., evidenciaron que el número de células no se ve afectado por estrés crónico, contrario a lo observado por Ulrich-Lai et $a l$, quienes señalaron que la exposición a estrés crónico variable, genera la aparición de hiperplasia en la parte externa e hipertrofia en la parte interna de la zona fasciculada de la GSR. Por otra parte, nuestros resultados, no mostraron diferencias estadísticamente significativas en la Nv entre los grupos C2, E1 y E2. Sin embargo, cuando la exposición a las experiencias adversas se presentaron en conjunto (E3), el número de células grandulares de la zona fasciculada de la GSR disminuyó significativamente, comparada con los otros grupos. Resultados similares fueron observado por Petrovic-Kosanovic et al., pese a las diferencias del tipo de estrés utilizado.

En el grupo control C2, se observaron aumentos de la Vv y de la Sv de la zona fasciculada, al compararlo con el grupo control C1. Estos hallazgos podrían tener relación con lo observado por Milosevic et al., quienes encontraron un aumento en el volumen celular de la zona fasciculada de ratas expuestas a estrés crónico por exposición a luz constante, lo que indicaría que el aumento de estos parámetros estereológicos podría deberse a una hipertrofia celular. Estos hallazgos, según Ulrich-Lai et al., reflejarían la alta demanda en la síntesis y secreción de corticosterona liberada en respuesta al sometimiento de estrés crónico.

Todos los parámetros estereológicos del grupo E1 fueron menores a los observado en el grupo control C2; sin embargo, las diferencias fueron significativas sólo para la Vv y la Sv. Estos resultados podrían sugerir que la experiencia adversa temprana por ruptura del vínculo social madre-cría, podría generar consecuencia en la actividad normal de la GSR durante el estrés crónico intermitente en la adultez. Este hecho, podría explicar en parte las observaciones de Toth et al., respecto a las alteraciones de los comportamientos social y emocional en ratas adul- 
VÁSQUEZ, B.; SANDOVAL, C.; SANHUEZA, C.; SILVA, J. \& DEL SOL, M. Efectos de las experiencias adversas temprana y tardía en las características morfométricas y estereológicas de la glándula suprarrenal de ratas Sprague Dawley sometidas a estrés durante su Vida adulta. Int. J. Morphol., 31(1):15-22, 2013.

tas expuestas a experiencias adversas en edades tempranas y un mayor perfil ansiogénico durante estados de estrés en la vida adulta, según Pascual et al.

El grupo E2 presentó resultados similares al grupo E1 en cuanto a la $\mathrm{Nv}$ al compararlo con el grupo C2. Se observó además que los valores de $\mathrm{Vv}$ fueron significativamente menores al del grupo E1. Estos resultados podrían indicar que los cambios morfológicos de la GSR en ratas también estarían asociados a la contigüidad de la experiencia adversa tardía por aislamiento con el estímulo estresor (tail pinch).

El grupo experimental E3, presentó los cambios estereológicos más evidentes en la zona fasciculada de las GSR. La Nv, Vv y $\mathrm{Sv}$ resultaron disminuidas significativamente respecto a todos los grupos e, inclusive, comparado con el grupo control $\mathrm{C} 1$, que no fue sometido a estrés $(p<0,001)$. Estos cambios apoyarían la hipótesis de que la GSR, bajo estas condiciones tendría menor actividad glandular, y por tanto, una desensibilización al estímulo de la ACTH, asociado a una baja producción de corticosterona. En relación a estos resultados, King \& Edwards (1999), encontraron altos niveles de ACTH, pero no de corticosterona en ratas alejadas de sus madres a los 21 días y posteriormente sometidas a estrés crónico durante la edad adulta, lo cual respaldaría los cambios estereológicos observados en el presente estudio. Estos resultados no concuerdan a lo señalado por Cruz y Malkesman et al., debido probablemente a que la privación social aplicada en el modelo experimental de estos autores, no estaba acompañada de la interrupción del vínculo madre-cría.

En conclusión, las experiencias adversas temprana y/o tardía a la que fueron expuestos los individuos de los grupos experimentales, juegan un rol importante respecto a los cambios estereológicos provocados en la GSR de ratas sometidas a estrés crónico en la vida adulta.

VÁSQUEZ, B.; SANDOVAL, C.; SANHUEZA, C.; SILVA, J. \& DEL SOL, M. Effects of early and late adverse experiences in morphometric and stereological characteristics of the adrenal gland of Sprague Dawley rats subjected to stress during adulthood. Int J. Morphol., 31 (1):15-22, 2013.

SUMMARY: The early break in maternal bonding and social isolation are variables involved with social and emotional behaviors and also with increased anxiety, especially in stressful situations. However, there is no research to explain the morphological changes of the adrenal gland (GSR). Therefore, our objective was to experimentally study through alteration model of social ties and early mother-cub bond alteration belated social isolation, the effect on the morphometric and stereological characteristics of the GSR in rats of the Sprague Dawley subjected to intermittent chronic stress in adulthood. We used 35 newborn female rats, divided into groups of 7 under lactating and standardized feeding conditions. The experimental groups were exposed to adverse experiences early (E1), late (E2), and early/late (E3) and subsequently, subject to intermittent chronic stress in adulthood. Left GSR was isolated from each animal determining morphometric characteristics and stereological parameters. The absolute weight was higher in control group C2 and the experimental group E1. In the group E3 number of cells per $\mathrm{mm}^{3}$, the percentage of glandular tissue and density of the surface in the fascicular area of the GSR was lower. Stereological characteristics of the GRS in rats, not only can be affected by exposure to stress in adulthood, but also early and / or late adverse experiences play an important role in the changes of quantitative morphological parameters observed in this gland.

KEYWORDS: Rat; Stress; Adverse experience; Adrenal gland.

\section{REFERENCIAS BIBLIOGRÁFICAS}

Alario, P.; Gamallo, A.; Beato, M. J. \& Trancho, G. Body weight gain, food intake and adrenal development in chronic noise stressed rats. Physiol. Behav., 40:29-32, 1987.

Bledsoe, A.; Oliver, K.; Scholl, J. \& Forsten, G. Anxiety states induced by post-weaning social isolation are mediated by $\mathrm{CRF}$ receptors in the dorsal raphe nucleus. Brain Res Bull., 85(34):117-22, 2011

Chrousos, G. P. Stress and disorders of the stress system. Nat. Rev. Endocrinol., 5:374-81, 2009.

Chrousos, G. P. \& Kino, T. Glucocorticoid action networks and complex psychiatric and/or somatic disorders. Stress, 10(2):213-9, 2007.

Cruz, D. B. Efecto de la privación social en la agresión y la ansiedad de ratas machos Wistar. Acta Colombiana de Psicología, 99:39-49, 2003.

Goldstein, D. \& Kopin, I. Evolution of concepts of stress. Stress, 10(2):109-20, 2007.

Herman, J.P.; Prewitt, C.M. \& Cullinan, W.E. Neuronal circuit regulation of the hypothalamo-pituitary-adrenocortical stress axis. Crit. Rev. Neurobiol., 10 (3-4):371-94, 1996. 
VÁSQUEZ, B.; SANDOVAL, C.; SANHUEZA, C.; SILVA, J. \& DEL SOL, M. Efectos de las experiencias adversas temprana y tardía en las características morfométricas y estereológicas de la glándula suprarrenal de ratas Sprague Dawley sometidas a estrés durante su Vida adulta. Int. J. Morphol., 31(1):15-22, 2013.

Katz, R. J. \& Roth, K. Tail pinch induced stress-arousal facilitates brain stimulation reward. Physiology \& Behavior, 22:193-4, 1979 .

King, J. A. \& Edwards, E. Early stress and genetic influences on hypotallamic-pitutary-adrenal axis functioning in adulthood. Horm. Behav., 36: 79-85, 1999.

Koko, V.; Djordjeviæ, J.; Cvijiæ, G. \& Davidoviæ, V. Effect of acute heat stress on rat adrenal glands: a morphological and stereological study. J. Exp. Biol., 207:4225-30, 2004.

Malkesman, O.; Maayan, R.; Wezman, A. \& Weller, A. Aggressive behavior and HPA axis hormones after social isolation in adult rats of two different genetic animal models for depression. Behavioural Brain Res., 175(2):408-14, 2006.

Milosevic, V.; Trifunovic, S.; Sekulic, M.; S`os`ic-Jurjevic, B.; Filipovic, B.; Negic, N.; Nestorovic, N.; ManojlovicStojanoski, M. \& Starc`evic, V. Chronic Exposure to Constant Light Affects Morphology and Secretion of Adrenal Zona Fasciculata Cells in Female Rats. Gen. Physiol. Biophys., 24:299-309, 2005.

Nadal, R. \& Armario, A. Mecanismos de susceptibilidad al estrés. Hipertens. Riesgo Vasc., 27(3):117-24, 2010.

Ojea, M.; Fustiñana, C. \& Argibay, P. Efectos del destete temprano sobre el comportamiento adulto en un modelo animal. Arch. Argent. Pediatr., 108(4):350-3, 2010.

Pascual, R. La interrupción temprana del vínculo social altera la organización citoarquitectónica y expresión de neuropéptidos en la corteza prefrontal. Rev. Chil. Neuropsiquiatr., 40(2):9-20, 2002.

Pascual, R.; Catalán, M. \& Fuentealba, M. Rasgos de ansiedad y alteraciones neuronales en la corteza prefrontal medial, ocasionadas por experiencias adversas tempranas. Rev. Chil. Neuro-psiquiat., 41(3):201-11, 2003.

Pellegrini, A.; Grieco, M.; Materazzi, G.; Gesi, M. \& Ricciardi, P. Stress-induced morphohistochemical and functional changes in rat adrenal cortex, testis and major salivary glands. Histochem J., 30 (10):695-701, 1998.

Petrovic-Kosanovic, D.; Velickovic, K.; Koko, V.; Jasnic, N.; Cvijic, G. \& Milosevic, M. Effect of acute heat stress on rat adrenal cortex-a morphological and structural study. Cent. Eur. J. Biol., 7(4): 611-9, 2012.

Scherle, W. A simple method for volumetry of organs in quantitative stereology. Mikroskopie, 26:57-63, 1970.

Toth, E.; Avital, A.; Leshem, M.; Richter-Levin, G. \& Braun, K. Neonatal and juvenile stress induces changes in adult social behavior without affecting cognitive function. Behav. Brain Res., 190:135-9, 2008.
Ulrich-Lai, I.; Figueiredo, H.; Ostrander, M.; Choi, D.; Engeland, W. \& Herman, J. Chronic stress induces adrenal hyperplasia and hypertrophy in a subregion-specific manner. Am. J. Physiol. Endocrinol. Metab,, 291:965-73, 2006.

Ziegler, D. R. \& Herman, J. P. Neurocircuitry of stress integration: anatomical pathways regulating the hypothalamo-pituitaryadrenocortical axis of the rat. Integ. Comp. Biol., 42:541-51, 2002.

Dirección para correspondencia:

Bélgica Vásquez Pastene

Universidad de Tarapacá

CHILE

Email:bvasquezp@uta.cl

Recibido : 02-01-2013

Aceptado: 11-02-2013 